\title{
Dyspnea, CTCAE
}

National Cancer Institute

\section{Source}

National Cancer Institute. Dyspnea, CT CAE. NCI Thesaurus. Code C58185.

A disorder characterized by an uncomfortable sensation of difficulty breathing. 